\title{
THE USE OF AUTOGENOUS AURICULAR CARTILAGE IN THE MANAGEMENT OF UPPER EYELID ENTROPION
}

\author{
AMER YAQUB and BRIAN LEATHERBARROW \\ Manchester
}

\begin{abstract}
SUMMARY
Upper eyelid entropion can occur as a consequence of inflammation, infection, trauma or surgery. It may very rarely occur as a congenital eyelid malposition. Numerous surgical procedures have been described to correct it depending on the primary anatomical and pathophysiological defects. We describe the use of autogenous auricular cartilage for its correction where the tarsal plate is found to be deficient. Seven patients were studied prospectively after correction of their upper eyelid entropion with autogenous auricular cartilage with a minimum follow-up of 6 months. We report the surgical technique, and the results and complications of the procedure.
\end{abstract}

Upper eyelid entropion is an eyelid malposition characterised by inversion of the eyelid margin with secondary misdirection of the eyelashes which, if untreated, can lead to severe ocular morbidity. The entropion can vary in severity and typically, in its early phase, may be misdiagnosed as simple trichiasis leading to inappropriate management. Trichiasis may, however, coexist. The entropion is usually acquired but may rarely be congenital. It is usually secondary to scarring and contracture of the posterior lamella following trachoma, Stevens-Johnson syndrome, cicatricial pemphigoid, chronic mucous plaque conjunctivitis complicating herpes zoster ophthalmicus, chronic blepharoconjunctivitis and chemical or thermal burns. ${ }^{1-3}$ It may also occur due to a congenital deformity of the tarsal plate or may be iatrogenic following excessive removal of tarsal plate during certain ptosis procedures. It is also a problem frequently encountered in anophthalmic patients with contracted sockets. In severe cases the entropion is commonly associated with lagophthalmos.

Correspondence to: Mr B. Leatherbarrow, FRCS, FRCOphth, Manchester Royal Eye Hospital, Oxford Road, Manchester M13 9WH, UK.
A number of procedures have been described to manage upper lid entropion based on various concepts. The choice of procedure depends on a number of factors: the degree and severity of the entropion, the thickness of the tarsal plate, the presence or absence of keratin on the posterior lamella, the presence and degree of lagophthalmos, and the nature of associated corneal disease. ${ }^{4}$ These procedures do not, however, take into consideration those situations in which an absence of a significant amount of tarsal plate alone is responsible for loss of the structural integrity of the upper eyelid. Such situations are not always complicated by severe posterior lamellar contracture requiring posterior lamellar grafts consisting of a mucosal surface. Such a circumstance may be encountered in patients following trauma, multiple previous operations on the eyelids and congenital entropion. Although the use of autogenous auricular cartilage in oculoplastic surgery is not new, ${ }^{5}$ its use is not widespread. We describe a surgical technique for the management of upper eyelid entropion in such situations using autogenous auricular cartilage to restore the structural integrity of the upper eyelid.

\section{MATERIAL AND METHODS}

We prospectively studied 8 patients with upper eyelid entropion in whom we had observed a loss of upper eyelid tarsus in the absence of posterior lamellar contracture. The aetiology of the entropion was recorded and any previous surgical treatment noted. The upper eyelid was everted and the vertical height of the tarsal plate recorded. The presence of keratin on the posterior lamella or a loss of the superior fornix were considered contraindications to this procedure. The patient was examined for any associated lagophthalmos and, if this was present, it was measured and recorded. Any associated corneal pathology was documented. The ears were examined to ensure that the pinna was normal and suitable for 


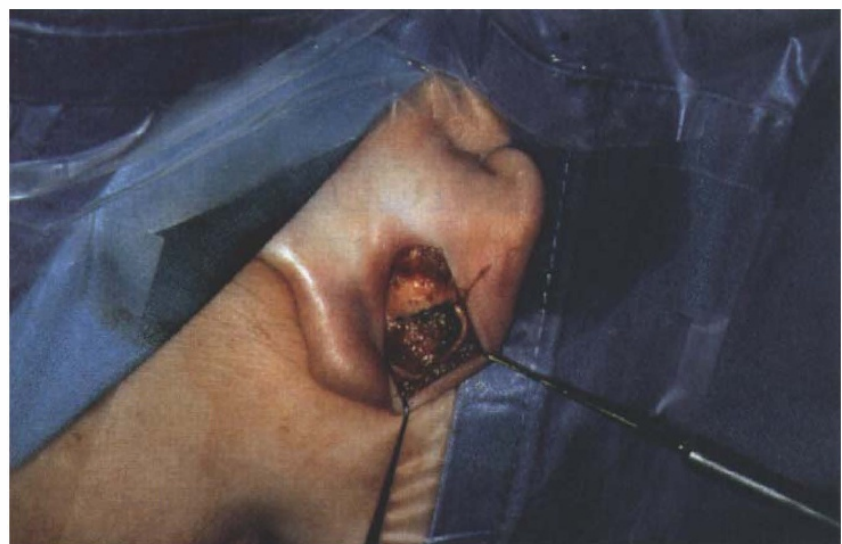

Fig. 1. The graft bed after harvesting the auricular cartilage.

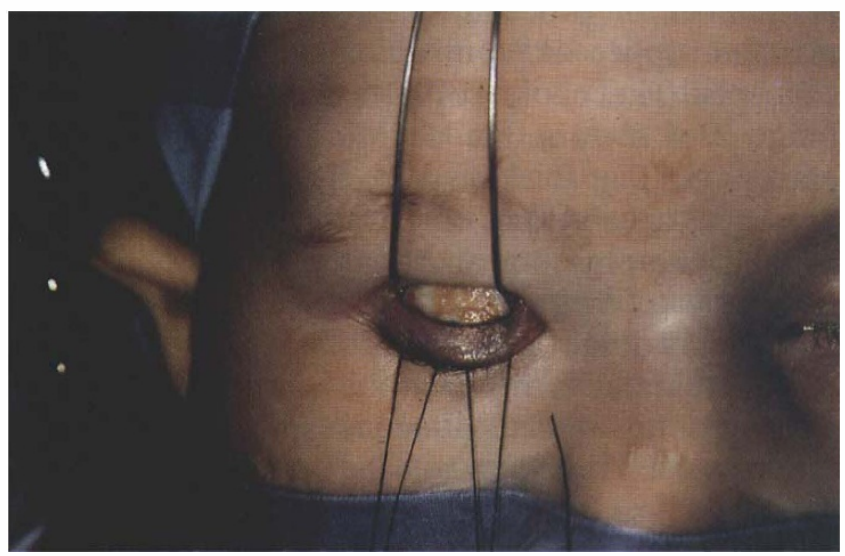

Fig. 3. Intraoperative photograph of the graft in place before the levator aponeurosis is sutured to its anterior surface.

harvesting auricular cartilage. The patients were all photographed pre-operatively. The surgery was performed by a single surgeon (B.L.). The patients were reviewed 1 week post-operatively and subsequently at 6 weeks, 3 months and 6 months postoperatively. At the 6 months post-operative visit the patients were again photographed. The position of the upper eyelid was noted and any complications recorded.

\section{Surgical Technique}

An upper eyelid skin crease incision is marked and 2 $\mathrm{ml}$ of $0.25 \%$ bupivicaine with 1:200 000 units of adrenaline mixed 50:50 with $2 \%$ lignocaine are injected subcutaneously into the upper eyelid. A further 3-5 $\mathrm{ml}$ of the same local anaesthetic solutions are injected subcutaneously on the posterior aspect of the pinna. An upper lid skin crease incision is made using a no. 15 Bard Parker blade and the orbicularis oculi muscle is dissected using blunttipped Westcott scissors to expose the tarsal plate remnant. This is then exposed horizontally and inferiorly until the lash roots are just visible creating a 'pocket'. The orbital septum is opened exposing the

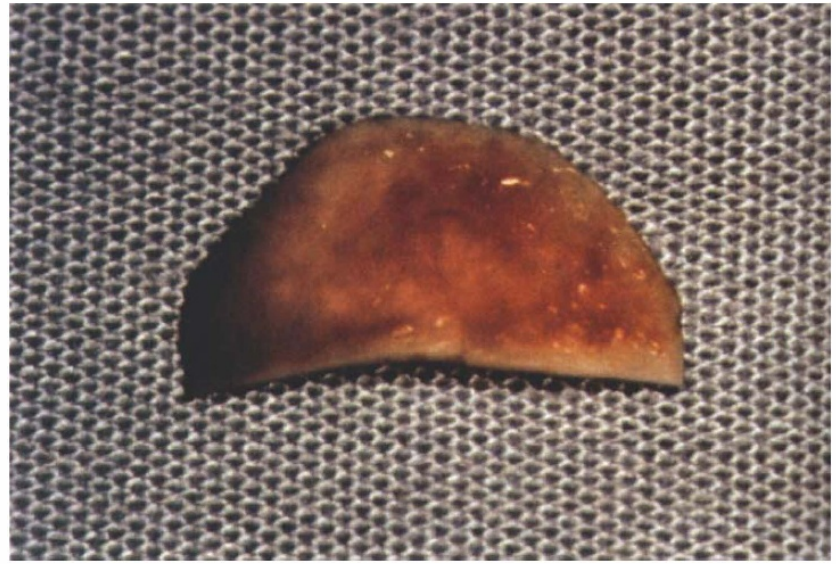

Fig. 2. An auricular cartilage graft shaped like a normal tarsal plate.

preaponeurotic fat pad and the underlying levator aponeurosis. This is carefully dissected off the tarsal plate. Next the underlying Müller's muscle is dissected from the underlying conjunctiva and recessed and any subconjunctival adhesions are freed.

Then non-crushing tissue clamps are applied to the margin of the ear to aid in retraction. A curvilinear incision is marked along the mid-portion of the pinna posteriorly. An incision is made using a no. 15 Bard Parker blade and the subcutaneous tissues are dissected from the underlying auricular cartilage using blunt-tipped Westcott scissors (Fig. 1). The required size of graft is measured from the eyelid and marked on the auricular cartilage in a configuration mimicking that of a normal tarsal plate (Fig. 2). A small incision is made through the auricular cartilage with a no. 15 blade and the dissection is completed using the Westcott scissors, taking care not to perforate the skin on the anterior surface of the pinna. The wound is closed using a continuous interlocking 4.0 nylon suture and, in order to prevent a subsequent haematoma, a dental roll bolster covered in Vaseline gauze is sutured in place on each side of the pinna.

The graft is cleaned and scored vertically with the no. 15 blade on one side to create a curve of the graft mimicking that of a normal tarsal plate. The graft is then placed into the eyelid wound within the dissected 'pocket' (Fig. 3). Müller's muscle is left unsutured at the upper border of the graft. The levator aponeurosis is sutured to the upper one-third of the graft using interrupted 7.0 vicryl sutures. The skin wound is closed using interrupted 7.0 vicryl sutures that incorporate the levator aponeurosis to re-create a skin crease. Topical antibiotic ointmentis liberally applied and a compressive dressing placed that is left in place for 1 week. The auricular sutures are removed on the first post-operative visit at 1 week. 


\section{RESULTS}

Seven patients underwent upper eyelid entropion surgery with placement of an autogenous auricular cartilage graft. Their details are summarised in Table I.

The ages of the patients ranged from 9 months to 90 years. The underlying causes of the entropion were varied. Patient 1, a 9-month-old infant, had a unilateral congenital entropion with no other eyelid or ocular abnormality (Fig. 4). The right tarsal plate was grossly deficient compared with the normal left side (Fig. 5). The cornea showed a widespread punctate epitheliopathy. No previous surgery had been performed.

Patients 2 and 3 were Asian immigrants with old trachomatous disease. Both patients had severely scarred corneas. Both had previously undergone upper eyelid surgery of an undetermined nature. Patient 3 underwent a penetrating keratoplasty on the left eye 4 weeks following the eyelid surgery.

Patient 4 had previously undergone a Fasanella Servat procedure for the management of a congenital ptosis under the care of a plastic surgeon some 15 years earlier. She had subsequently undergone multiple cryotherapy treatments for secondary 'trichiasis'. She had resorted to repeated self-epilation of residual lashes.

Patient 5 was anophthalmic with a mild socket contracture and an orbital volume deficit. He had undergone a secondary orbital implant procedure 3 months earlier.

Patient 6 had suffered a severe molten metal injury to his right eye. He had previously undergone multiple eyelid operations.

Patient 7 was anophthalmic having suffered trauma to the eyelids and socket and had undergone multiple operations.

The follow-up ranged from 6 months to 1 year. In all cases the surgical procedure described above was successful in repositioning the eyelid margin with no complications (Fig. 6). No patient developed a secondary ptosis or eyelid retraction. Patient 2 underwent cryotherapy 2 months post-operatively for the treatment of lateral upper eyelid trichiasis. None of the other patients required any further eyelid procedure. The donor sites healed well, leaving a cosmetically imperceptible localised thinning of the pinna.

\section{DISCUSSION}

Cicatricial entropion of the upper eyelid can result from a variety of disorders causing anatomical and pathophysiological defects. Numerous procedures have been described based on the following concepts: $^{6-19}$ (1) repositioning the anterior lamella forwards to pull the lashes away from the globe; (2) splitting the lid margin alone or combined with grafts; (3) lengthening of the posterior lamella with composite grafts of tarsoconjunctiva, nasal septum, epiglottis; (4) lengthening of the posterior lamella with materials without a mucous membrane such as sclera, collagen film or chondroplast; and (5) excision of the tarsal plate.

The most frequent cause of upper eyelid entropion is scarring and contracture of the posterior lamella of the eyelid. This usually results in inversion of the lid margin and trichiasis. In a vast majority of these patients, especially trachoma patients, the tarsal plate is thickened and there is no lagophthalmos. Most procedures designed to correct the entropion depend on this abnormality, i.e. a thickened and scarred tarsal plate. We have come across a number of patients who, either primarily or consequent to previous surgery, have developed atrophy of the tarsal plate resulting in poor structural integrity of the entire eyelid and instability of the eyelid margin. These patients do not have a firm tarsal plate that could be used to anchor and rotate the eyelid margin away from the globe.

Most of the procedures previously described to correct such cases either consist of excising the tarsal remnant with recession of lid retractors or alternatively use a posterior lamellar graft employing various materials such as tarsoconjunctival graft, nasal septal graft, hard palate graft, conchal graft, epiglottis or implantation of chondroplast. All these procedures use the conjunctival approach which, in addition to being technically difficult, should be avoided in patients with active cicatrising conjunctival inflammation seen in ocular surface disorders such as cicatricial pemphigoid or Stevens-Johnson syndrome.

Table I. Patient details

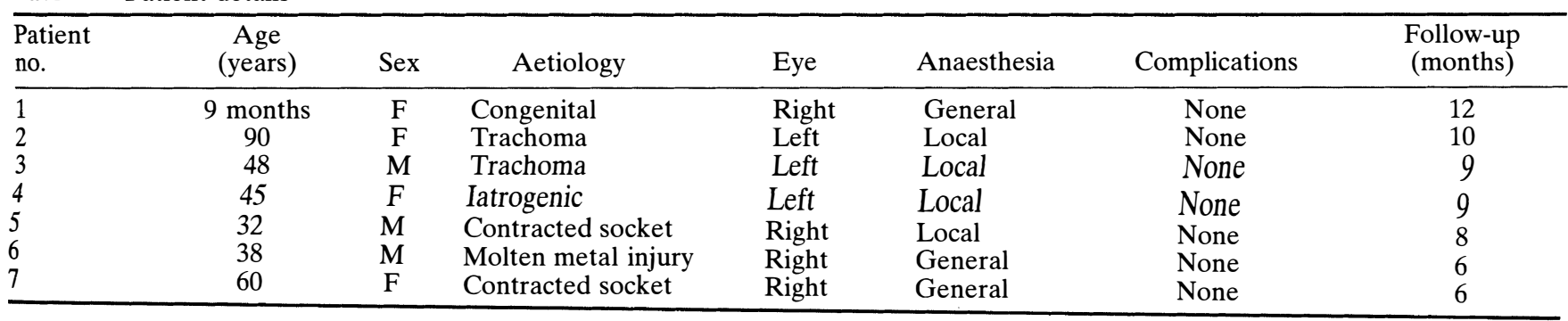




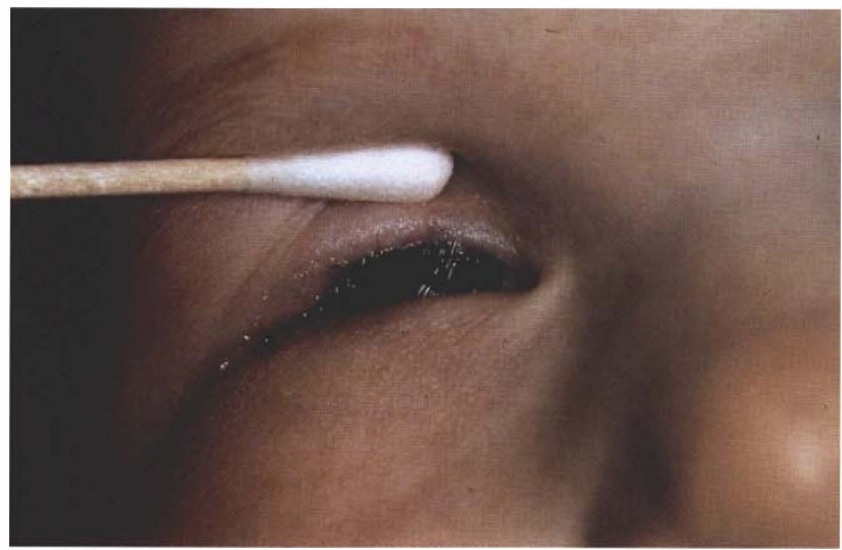

(a)

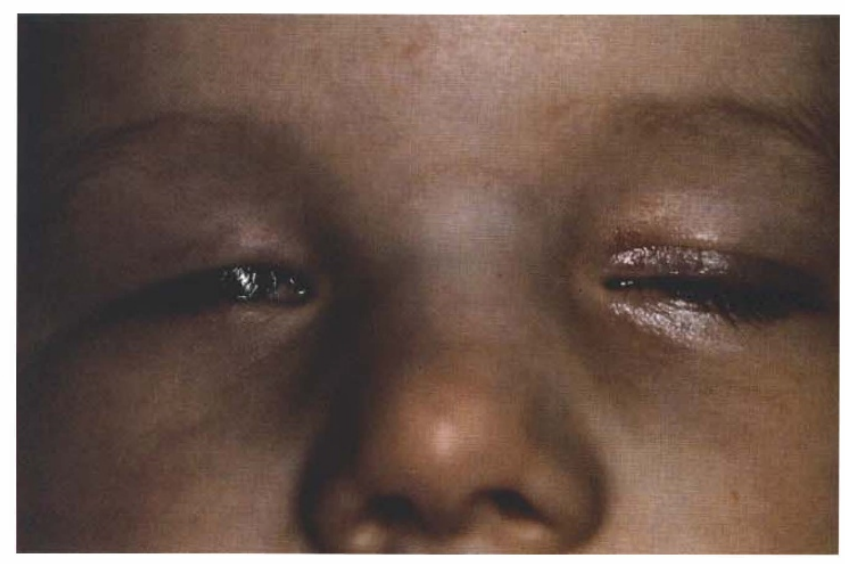

(b)

Fig. 4. Patient 1. (a), (b) Congenital upper eyelid entropion: pre-operative appearance.

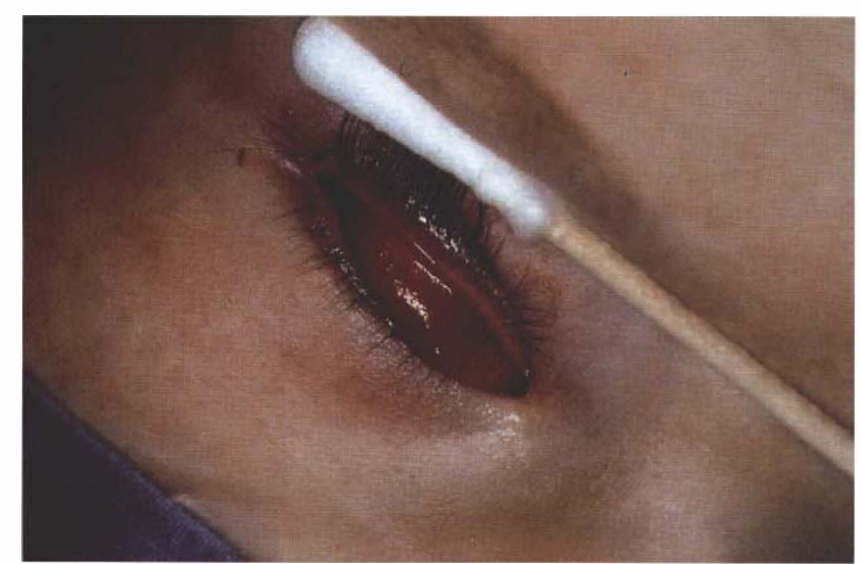

(a)

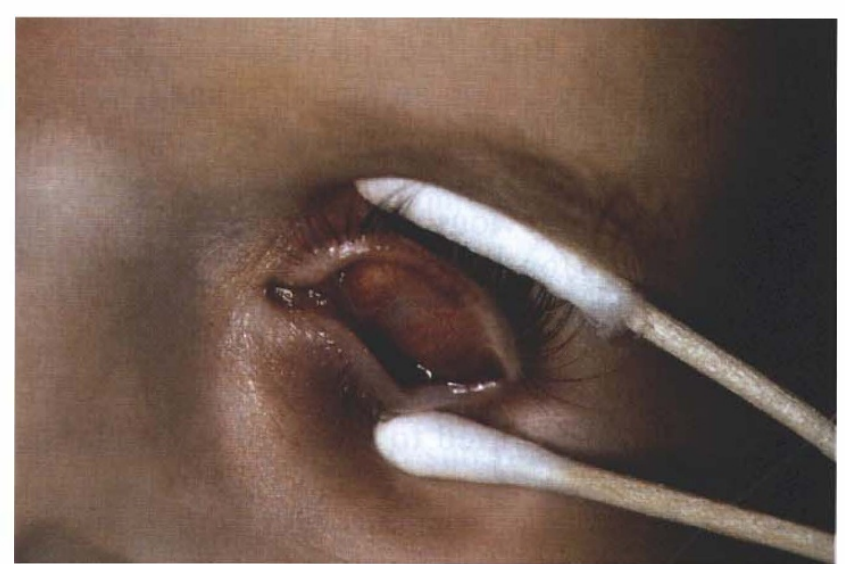

(b)

Fig. 5. Patient 1. (a) The tarsal plate of the eyelid with entropion. (b) The tarsal plate on the normal side.

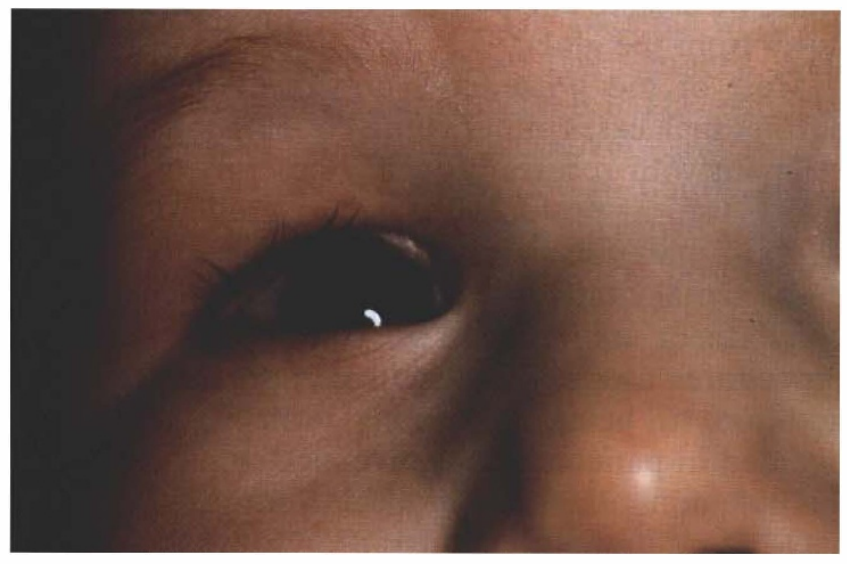

(a)

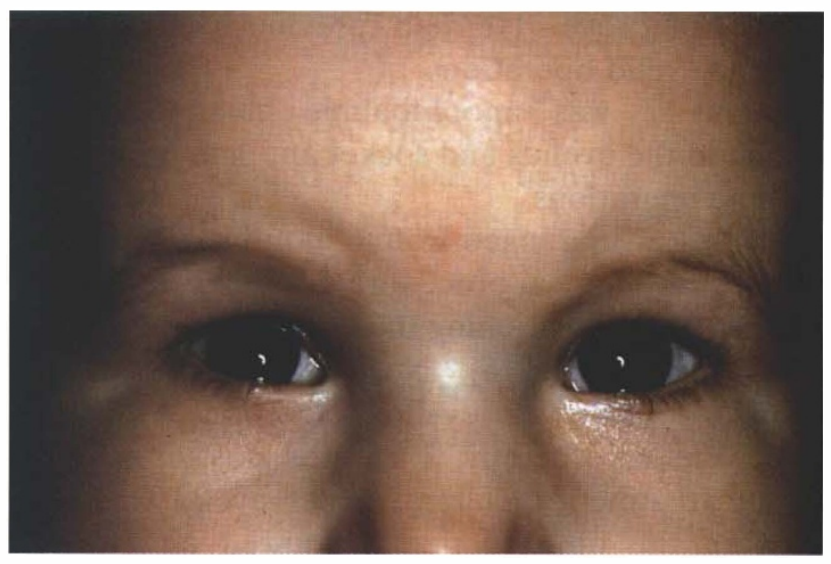

(b)

Fig. 6. Patient 1. (a), (b) Post-operative appearance after correction with an autogenous auricular cartilage graft.

The surgical technique we describe uses a cutaneous approach and essentially avoids incision of the conjunctiva. It gives structural support to the eyelid and provides a strut against which the eyelid margin can be rotated away from the globe. The auricular cartilage is easy to harvest and does not result in any appreciable deformity of the pinna. This procedure is particularly useful in patients in whom subsequent corneal grafting is planned.

The surgical technique we describe is effective in correcting cicatricial upper eyelid entropion in patients with lid retraction and tarsal atrophy. It achieves rotation of the eyelid margin away from the eye as well as providing a firm support to the eyelid. 
It is important to ensure that the superior fornix is not compromised, that lagophthalmos does not exceed $2-3 \mathrm{~mm}$ on gentle eyelid closure and that there is no keratin present on the posterior lamella before selecting this procedure for the management of patients with upper eyelid entropion.

Key words: Auricular cartilage, Entropion, Tarsal plate.

\section{REFERENCES}

1. Collin JRO, Kemp EG. Surgical management of upper lid entropion. Br J Ophthalmol 1986;70:575.

2. Dryden RM, Doxanas MT. Eyelid malposition. II. Ectropion and entropion. In: McCord CJ, editor. Oculoplastic surgery. New York: Raven Press, 1981: 145.

3. Rathbun JE. Entropion. In: Hornblass A, editor. Oculoplastic, orbital and reconstructive surgery. Vol 1: Eyelids. Baltimore: Williams \& Wilkins, 1988:322.

4. Collin JRO. A manual of systematic eyelid surgery. 2nd ed. Edinburgh: Churchill Livingstone, 1989:15.

5. Baylis HI, Rosen N, Neuhaus RW. Obtaining auricular cartilage for reconstructive surgery. Am J Ophthalmol 1982;93:709.

6. Dortzbach RK, Callahan A. Repair of cicatricial entropion of upper eyelids. Arch Ophthalmol 1971; 85:82.

7. Flanagan JC. Eye bank sclera in oculoplastic surgery. Ophthalmic Surg 1974;5:45.

8. Rubenzik R, Tenzel RR, Miller GR. Repair of cicatricial entropion of the upper eyelid. Am J Ophthalmol 1975;80:302.
9. Dryden RM, Soll DB. The use of scleral transplantation in cicatricial entropion and eyelid reconstruction. Trans Am Acad Ophthalmol Otolaryngol 1977;83:669.

10. Schmidt T, Leipert KP, Fellbaum C. Tarsusplastik mit Chondroplast (tarsusplasty with chondroplast). Fortschr Ophthalmol 1991;88:279.

11. Leone CR Jr. Mucous membrane grafting for cicatricial entropion. Ophthalmic Surg 1974;5:24.

12. Holt JE, Holt GR, van Kirk M. Use of temporalis fascia in eyelid reconstruction. Ophthalmology 1984; 91:89.

13. Mehrotra ON. Repairing defects of the lower eyelid with a free chondromucosal graft. Plast Reconstr Surg 1977;59:689.

14. Adams JL, Olson NR, Siders DB. The use of the epiglottis as an autologous composite graft in eyelid reconstruction. Ophthalmic Plast Reconstr Surg 1993; 9:206.

15. Baylis HI, Hamako C. Tarsal grafting for correction of cicatricial entropion. Ophthalmic Surg 1979;10:42.

16. Shorr N, Christenbury JD, Goldberg RA. Tarsoconjunctival grafts for upper eyelid cicatricial entropion. Ophthalmic Surg 1988;19:316.

17. Silver B. The use of mucous membrane from the hard palate in the treatment of trichiasis and cicatricial entropion. Ophthalmic Plast Reconstr Surg 1986;2:129.

18. Reacher MH, Munoz B, Alghassany A, et al. A controlled trial of surgery for trachomatous trichiasis of the upper lid. Arch Ophthalmol 1992;110:667.

19. Meyer-Rusenberg HW, Hoffman T, Emmerich KH. Ear cartilage as tarsus replacement. Fortschr Ophthalmol 1990;87:99-104. 